\title{
ECOLOGIE DES PRINCIPAUX INVERTEBRES FILTREURS DE LA BASSE NIVELLE (PYRENEES-ATLANTIQUES) I. SIMULIIDAE (DIPTERA, NEMATOCERA)
}

\author{
par A. NEVEU ${ }^{1}$ et L. LAPCHIN²
}

L'étude des imagos et des larves de Simuliidae a été effectuée au cours de trois années dans un cours d'eau de l'ouest des Pyrénées.

La température élevée, les herbiers à renoncules, une certaine pollution organique, sont les facteurs favorables au développement des Wilhelmia, surtout de $W$. lineata, associée à $W$. equina et $W$. mediterranea.

Les cyoles de développement sont synchrones, avec quatre générations par an. La génération d'été est la plus forte, des autres sont réduites par les crues et les parasites.

La taille des adultes suit les fluctuations thermiques.

Les stades larvaires présentent des différences dans leur microrépartition. La production larvaire est de l'ordre de $0,6 \mathrm{~g} / \mathrm{cm}^{3} / \mathrm{an}$ sur les renoncules et de $0,9 \mathrm{~g} / \mathrm{m}^{2} /$ an sur les cailloux, avec des rapports $\mathrm{P} / \overline{\mathrm{B}}$ annuels entre 6,6 et 7,6 .

\section{Ecology of the principal filter-feeding invertebrates of the Basse Nivelle (Atlantic Pyrenees). I. Simuliidae (Diptera : Nematocera).}

A study of adults and larvae of Simuliiidae was made over three years in waterbodies of the Western Pyrenees.

High temperature, stands of Ranunculus, and some organic pollution are favourable factors for the development of Wilhelmia, especially $W$. lineata, associated with $W$. equina and $W$. mediterranea.

The development cycles are synchronous with four generations per year. The summer generation is the most prolific, the others are reduced by floods and parasites.

The size of the adults follows fluctuations in temperature.

The larval stages show diffenences in their microdistribution. Larval production is of the order of $0.6 \mathrm{~g} / \mathrm{cm}^{3} /$ year on the Ranunculus and $0.9 \mathrm{~g} / \mathrm{m}^{2} /$ year on the stones, with annual $\mathrm{P} / \overline{\mathrm{B}}$ ratios between 6.6 and 7.6.

\section{1. - INTRODUCTION}

La Nivelle, petit fleuve côtier des Pyrénées-Atlantiques, se jette à la mer à Saint-Jean-de-Luz, après un cours de $40 \mathrm{~km}$; son bassin versant couvre environ $240 \mathrm{~km}^{2}$.

1. Laboratoire d'Ecologie Hydrobiologique, E.N.S.A., 65, rue de Saint-Brieuc, 35042 Rennes Cedex.

2. Station de Zoologie, I.N.R.A., B. P. 78, 06602 Antibes. 
Une série de travaux sur le macrobenthos est effectuée depuis 1973 sur la basse vallée. Ces nouvelles données complètent celles obtenues depuis plusieurs années sur un affluent, le Lissuraga (Benech 1971 ; Thibault 1971 a, b ; Neveu 1973 a, b, c ; Tiberghien 1976).

Deux familles sont particulièrement abondantes dans le benthos de la basse Nivelle : les Simuliidae et les Hydropsychidae. Ces invertébrés présentent deux caractères communs : ils vivent relativement fixés sur le substrat et consomment essentiellement des matières organiques dérivantes, très abondantes dans ce type de cours d'eau.

Cette première partie concerne l'étude des Simuliidae qui se développent abondamment dans les herbiers à Ranunculus sp. Leurs populations sont polyvoltines, les émergences ont lieu toute l'année et il est difficile de séparer les diverses générations. La densité du peuplement reste très variable suivant l'importance des crues qui arrachent les herbiers. C'est une nourriture de base, à certaines époques, pour les juvéniles de saumon.

\section{2. - TECHNIQUES ET METHODES D'ETUDE}

Une première étude taxinomique et phénologique des adultes a été effectuée à l'aide d'un piège lumineux de 200 watts, relevé 2 fois par semaine de février 1972 à mars 1973.

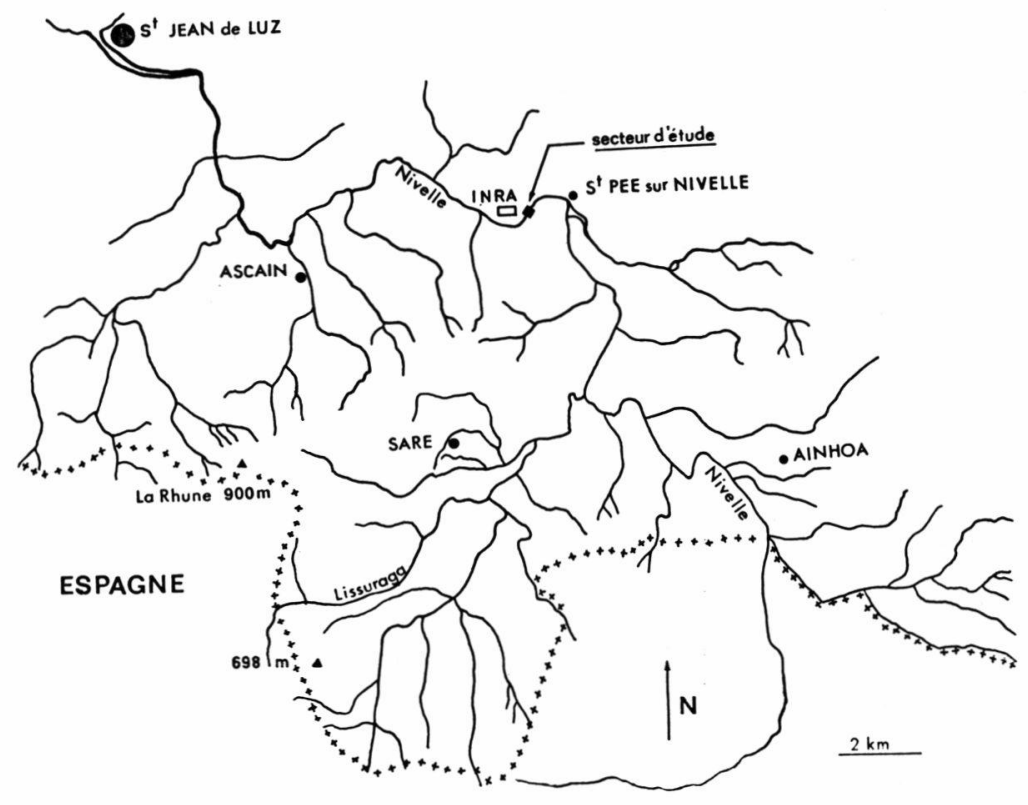

FIG. 1. - Bassin de la Nivelle. 
Les larves de simulies étant des animaux rhéophiles par excellence, les échantillons benthiques ont été prélevés uniquement dans les zones de courant d'un secteur bien délimité situé à $12 \mathrm{~km}$ de l'estuaire (fig. 1 et 2). Ces relevés ont été effectués à l'aide d'un filet de Surber prélevant $0,1 \mathrm{~m}^{2}$ et avec des mailles de $0,25 \mathrm{~mm}$ d'ouverture, semblable à celui de nos précédentes études.

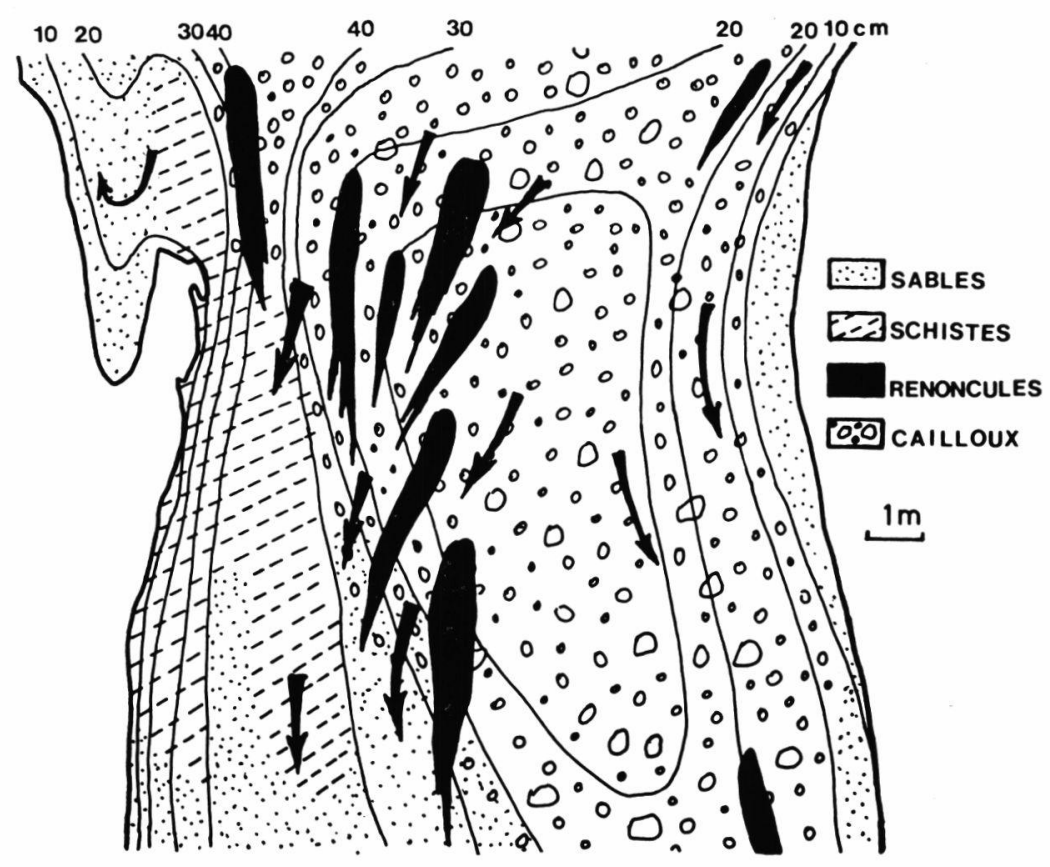

FIG. 2. - Secteur d'étude (distance des isobathes $=10 \mathrm{~cm}$ ).

Chaque mois, de mars 1973 à juin 1974, trois échantillons de 0,1 m² ont été prélevés dans les zones lotiques constituées par des amas de cailloux (de 10 à $15 \mathrm{~cm}$ ) dont les interstices sont colmatés par un sable grossier. Ces trois échantillons sont ensuite rassemblés et fixés au formol. Simultanément, des prélèvements ont été effectués dans les herbiers à renoncules en plaçant le filet de Surber en aval et en coupant avec des ciseaux quelques tiges à différents niveaux, ceci pour homogénéiser l'échantillon.

L'analyse détaillée de la composition faunique, des cycles de développement des autres groupes et de la structure du peuplement a été effectuée par ailleurs (Neveu et Vignes, sous presse). 


\section{3. - LE MILIEU}

Les caractéristiques du milieu "Nivelle » ont été étudiées en détail par ailleurs, elles peuvent se résumer brièvement (Lapchin et Neveu, sous presse).

Le climat conjugue une forte pulviosité annuelle $(1700 \mathrm{~mm})$ et de violents orages d'été. La température de l'eau est relativement élevée avec des maximums pouvant atteindre $23^{\circ} \mathrm{C}$. Au cours de la période d'étude du benthos, la température moyenne hebdomadaire est restée
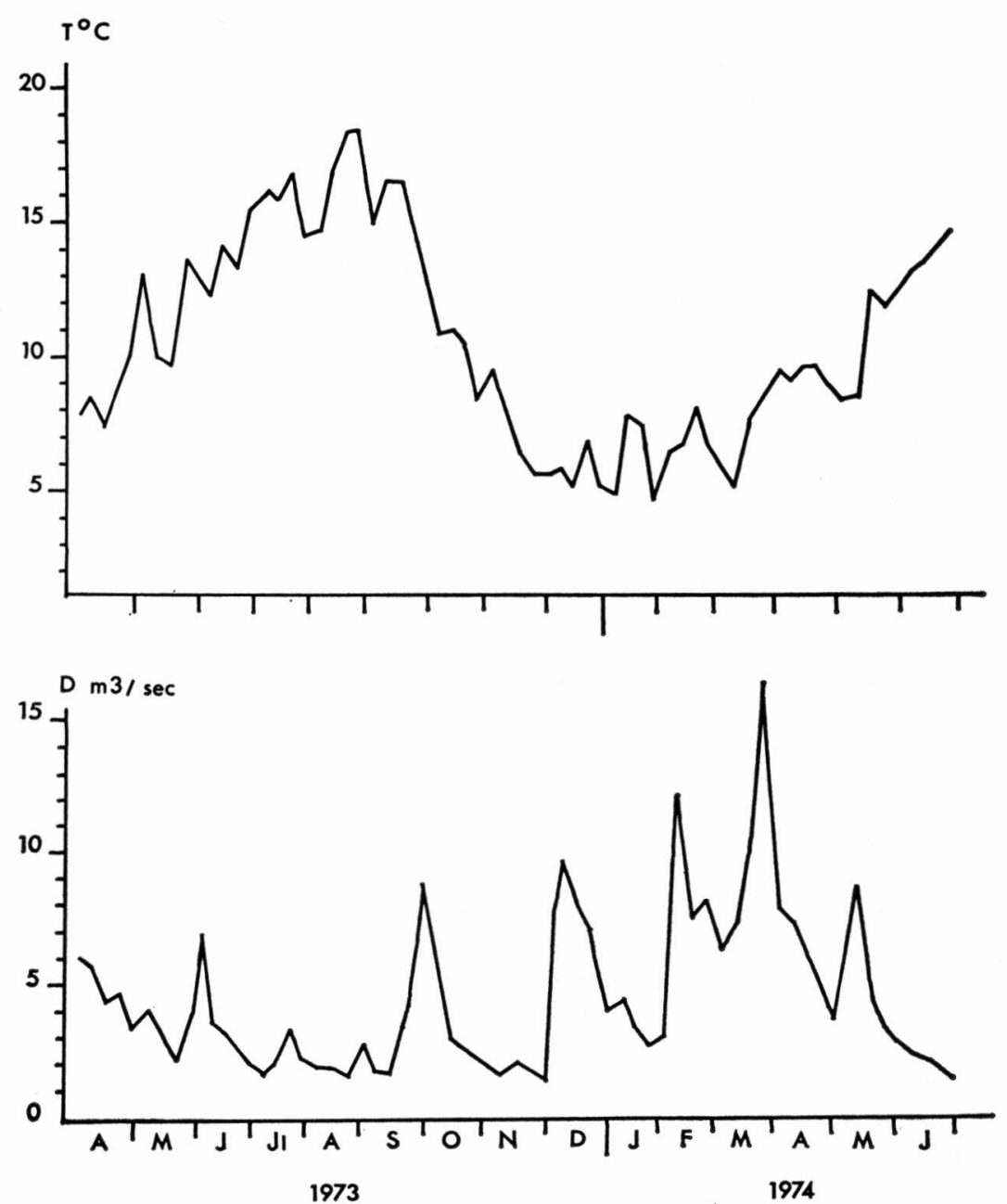

Fic. 3. - Caractéristiques du milieu au cours de l'étude du benthos de 1973 à 1974: température moyenne hebdomadaire de l'eau $\left(\mathrm{T}^{\circ} \mathrm{C}\right)$ et débit moyen hebdomadaire (D.). 
comprise entre $6,5^{\circ}$ et $21,2^{\circ}$; l'hiver, elle est de 8 à $9^{\circ}$ et l'été, de 17 à $18^{\circ}$ (fig. 3).

Les crues sont soudaines et violentes, les étiages prononcés, le rapport entre le débit maximum et l'étiage peut atteindre 240. En liaison avec ces crues, la transparence de l'eau est médiocre les deux tiers de l'année, par la présence d'argiles colloïdales issues de la décomposition des schistes de la haute vallée.

L'analyse chimique met en évidence une po!lution insidieuse, en relation avec les activités agricoles et touristiques, au niveau des nitrites $\left(0,10\right.$ à $0,35 \mathrm{mg} / 1$ de $\left.\mathrm{NO}_{2}{ }^{-}\right)$, des sels ammoniacaux $(0,06$ à $0,27 \mathrm{mg} / \mathrm{l}$ de $\left.\mathrm{NH}_{4}^{-}\right)$et des phosphates $(0,23 \mathrm{à} 1,38 \mathrm{mg} / \mathrm{l})$.

Avec une largeur de 10 à $15 \mathrm{~m}$, une pente de $1,5 \mathrm{p}$. 1000 , le secteur d'étude se trouve dans la zone à barbeau de Huet (1949). lI est constitué pour 50 p. 100 de sa surface par des cailloux, pour 37 p. 100 de sables et de limons, pour 7 p. 100 de la roche mère schisteuse et pour 6 p. 100 de cailloux recouverts par de renoncules (fig. 2). Mais cette dernière estimation dépend de la saison.

\section{4. - ETUDE DES CAPTURES IMAGINALES}

Nous avons suivi la nomenclature de Davies $(1966,1968)$ et surtout celle plus récente de Zwick (1974), il faut remarquer à ce sujet que Simulium salopiense Edw. est synonyme de Wilhelmia lineata $\mathrm{Mg}$. et que Simulium monticola sensu Doby et Rault (1960) de nos précédentes études (Neveu 1973 a) est synonyme de Simulium rheophilum Knoz.

\subsection{Diversité spécifique}

D'après les données de Davies $(1966,1968)$, il est possible de distintinguer les imagos mâles de Wilhelmia lineata $\mathrm{Mg}$. et de W. equina L., mais pratiquement impossible de séparer ceux du $W$. mediterranea Puri ; il en est de même des imagos femelles des trois espèces. C'est pourquoi, pour avoir une meilleure idée de l'importance respective des différentes espèces, il est utile de considérer avant tout la composition nymphale moyenne : W. lineata $\mathrm{Mg}$. (75,01 p. 100), W. equina $\mathrm{L}$. $(13,71$ p. 100$)$, Odagmia ornata $\mathrm{Mg}$. (9,12 p. 100), W. mediterranea Puri (1,98 p. 100), Eusimulium aureum Fries (0,05 p. 100), Simulium rheophilum Knoz (0,03 p. 100), S. variegatum Mg. (0,03 p. 100), S. reptans L. $(0,02$ p. 100$)$.

Sur 698 adultes capturés (409 $\hat{0}, 289$ \&), 632 sont Wilhelmia, le reste est constitué de : O. ornata (6 p. 100), S. variegatum (2,1 p. 100), E. aureum $(0,7$ p. 100$)$, E. latipes Mg. $(0,3$ p. 100), S. rheophilum $(0,1 \mathrm{p}$ 
100), E. angustitarse Lund. (0,1 p. 100). Pour les Wilhelmia, il y a 298 mâles de $W$. lineata contre 83 pour $W$. equinum + mediterranea et 251 femelles pour les trois espèces. En supposant que les mâles des deux dernières espèces se répartissent proportionnellement à leurs nymphes et qu'il en est de même pour des femelles, on obtient: $W$. lineata $(70,7$ p. 100), W. equina $(17,3$ p. 100$)$ et $W$. mediterranea $(2,4$ p. 100).

En ce qui concerne le sex-ratio, il faut remarquer que si les mâles de Wilhelmia sont plus abondants que les femelles $(R=1,52)$, pour les autres espèces c'est l'inverse: $O$. ornata $(\mathrm{R}=0,83), S$. variegatum $(\mathrm{R}=0,50)$.

Parmi les 289 femelles, seulement 17 présentent des œufs bien développés et 5 sont gorgées de sang, on peut donc considérer que 267 sont des adultes fraîchement éclos.

\subsection{Période de vol des différentes espèces}

L'examen des captures globales (fig. 4) montre trois périodes de vol d'importance décroissante: juillet-août, octobre-novembre, janvier-février.

Les différentes périodes apparaissent très nettement chez les mâles de $W$. lineata avec aussi quelques individus en avril-mai, il y aurait alors quatre générations; il semble en être de même pour $O$. ornata (fig. 5). Par contre, il n'y a pas d'imagos mâles de $W$. equina + mediterranea en avril-mai, les autres périodes de captures sont les mêmes que les précédentes. En ce qui concerne les femelles de Wilhelmia, l'on note quatre périodes d'émergence, les femelles d'automne sont relativement abondantes par rapport à celles d'été, alors que c'est l'inverse pour les mâles.

\subsection{Influence du climat sur l'importance des vols}

Le maximum des captures se situe en juillet-août, ce qui correspond au maximum de la température de l'air (fig. 4). A mesure que la saison s'avance, l'importance des vols diminue: la génération d'été est la plus abondante, celle du printemps suivant la plus faible. A remarquer que les températures de l'eau et de l'air restent suffisamment élevées l'hiver pour permettre une émergence imaginale et une activité de vol. La pluviosité ne semble pas avoir d'effet notable sur l'importance des vols, comme le remarque Carlsson (1967).

\subsection{Variations saisonnières de la taille}

Dans une précédente étude, nous avons montré que chaque généra- 


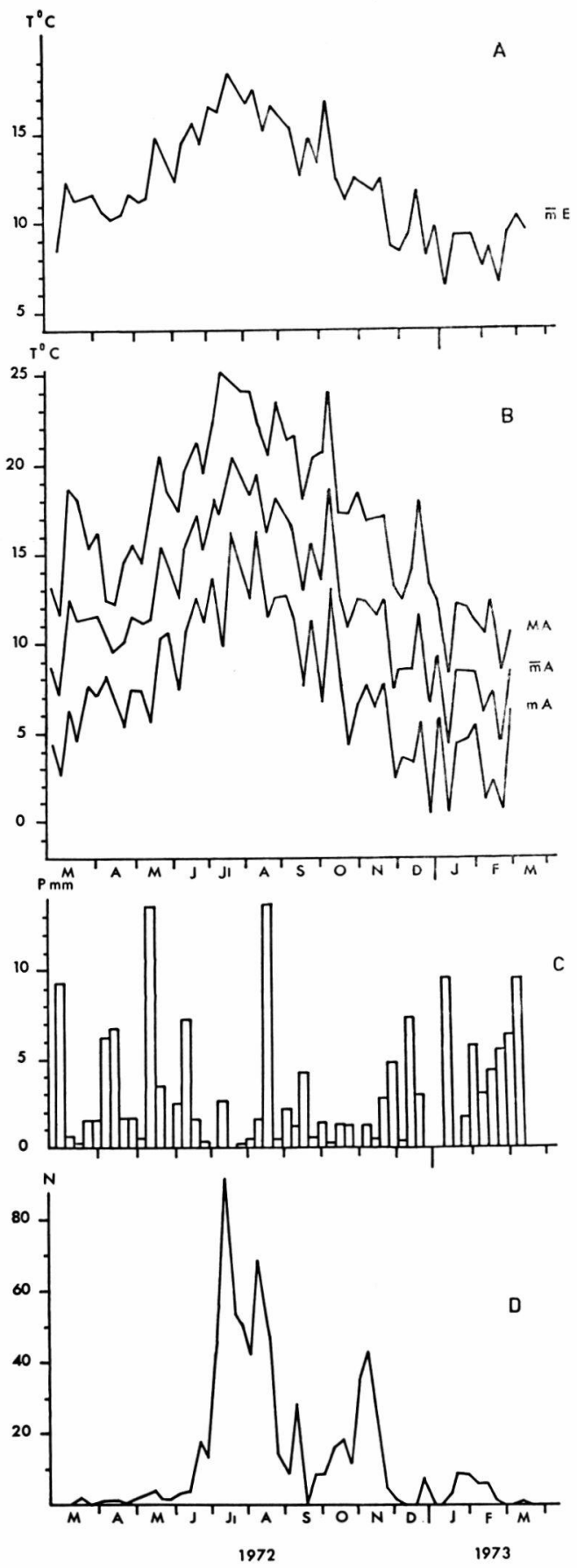

Fig. 4. - Caractéristiques climatiques au cours des captures au piège lumineux de 1972 à 1973 à Saint-Pé-sur-Nivelle. A) Température moyenne hebdomadaire de l'eau de la Nivelle; B) Température hebdomadaire de l'air: moyenne (mA), minimum des minimums (mA), maximum des maximums (MA); C) Pluviométrie hebdomadaire; D) Captures hebdomadaires de Simuliidae. 


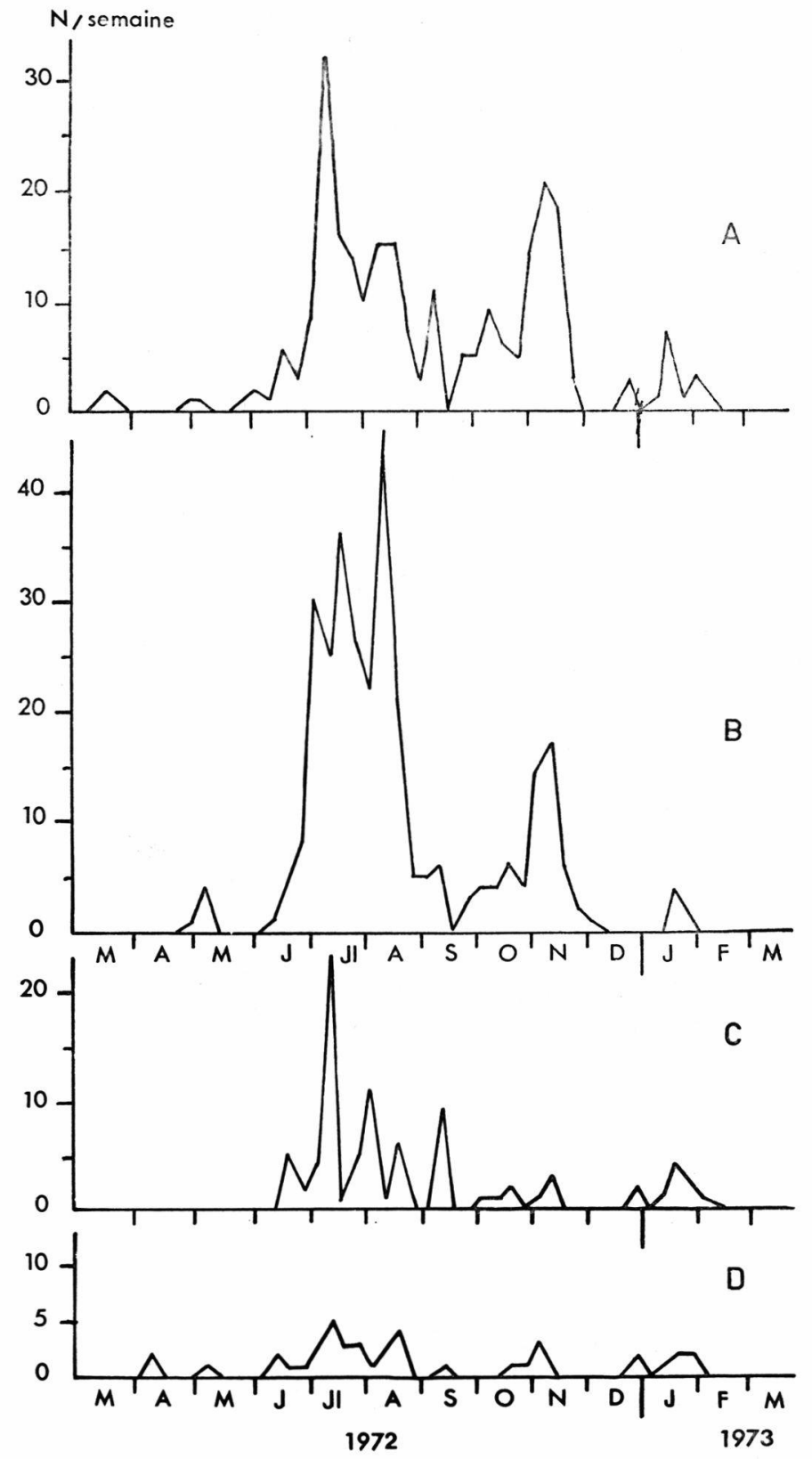

Fig. 5. - Captures hebdomadaires au piège lumineux des différentes espèces de Simuliidae. A) Femelles de Wilhelmia sp.; B) Mâles de W. lineata; C) Mâles de $W$. equina et $W$. mediterranea; D) Mâles et femelles de O. ornata. 
tion des Simuliidae du Lissuraga est constituée d'adultes ayant une taille moyenne particulière (Neveu $1973 \mathrm{a}$ et $\mathrm{b}$ ). Ce phénomène se retrouve dans la Nivelle au niveau des différentes espèces (fig. 6). Les coefficients de variation du basitarse sont du même ordre de grandeur que sur le Lissuraga, ce qui correspond à un rapport de l'ordre de trois entre la biomasse des plus petits adultes et celle des plus grands (Neveu 1973 a).

Les fluctuations de la taille suivent parfaitement celles de l'inverse de la température moyenne de l'eau. La petite taille estivale étant liée aux plus hautes températures, mais aussi à des conditions trophiques moins bonnes : raréfactions des diatomées, des particules organiques et étiage prononcé.

On peut donc conclure, à partir de l'intensité des émergences et des variations biométriques, que les quatre espèces les plus abondantes abondantes de la Nivelle présentent quatre générations par an. L'importance numérique de ces générations décroît régulièrement de l'été au printemps suivant. La taille moyenne des imagos est différente à chaque génération et corrélée au niveau thermique moyen correspondant.

Les autres espèces sont trop rares pour une étude de leur cycle, on peut noter cependant que $S$. variegatum a été capturée en juillet-août, en octobre-novembre et janvier-février, donc avec au moins trois générations, en concordance avec celles de Wilhelmia. On peut donc raisonnablement penser à une quatrième génération en avril-mai. $S$. aureum n'a été capturée qu'en automne, mais cela est en rapport avec la rareté de cette espèce et donc une faible probabilité de capture.

\section{5. - ETUDE DES POPULATIONS LARVAIRES ET NYMPHALES}

Les émergences imaginales étant synchronisées, on peut admettre que le développement larvaire l'est aussi et comme les larves d'O. ornata n'ont jamais dépassé 10 p. 100 d'un échantillon, il est possible d'étudier simultanément toutes les espèces. Par ailleurs, il n'est pas possible de séparer les larves des différentes espèces de Wilhelmia.

\subsection{Les populations nymphales}

Les récoltes de nymphes se font surtout sur les renoncules. Il s'agit surtout de Ranunculus fluitans Lmk, avec quelques rares Ranunculus aquatilis Linné.

Au cours de l'année, la densité des nymphes présente quatre pics 


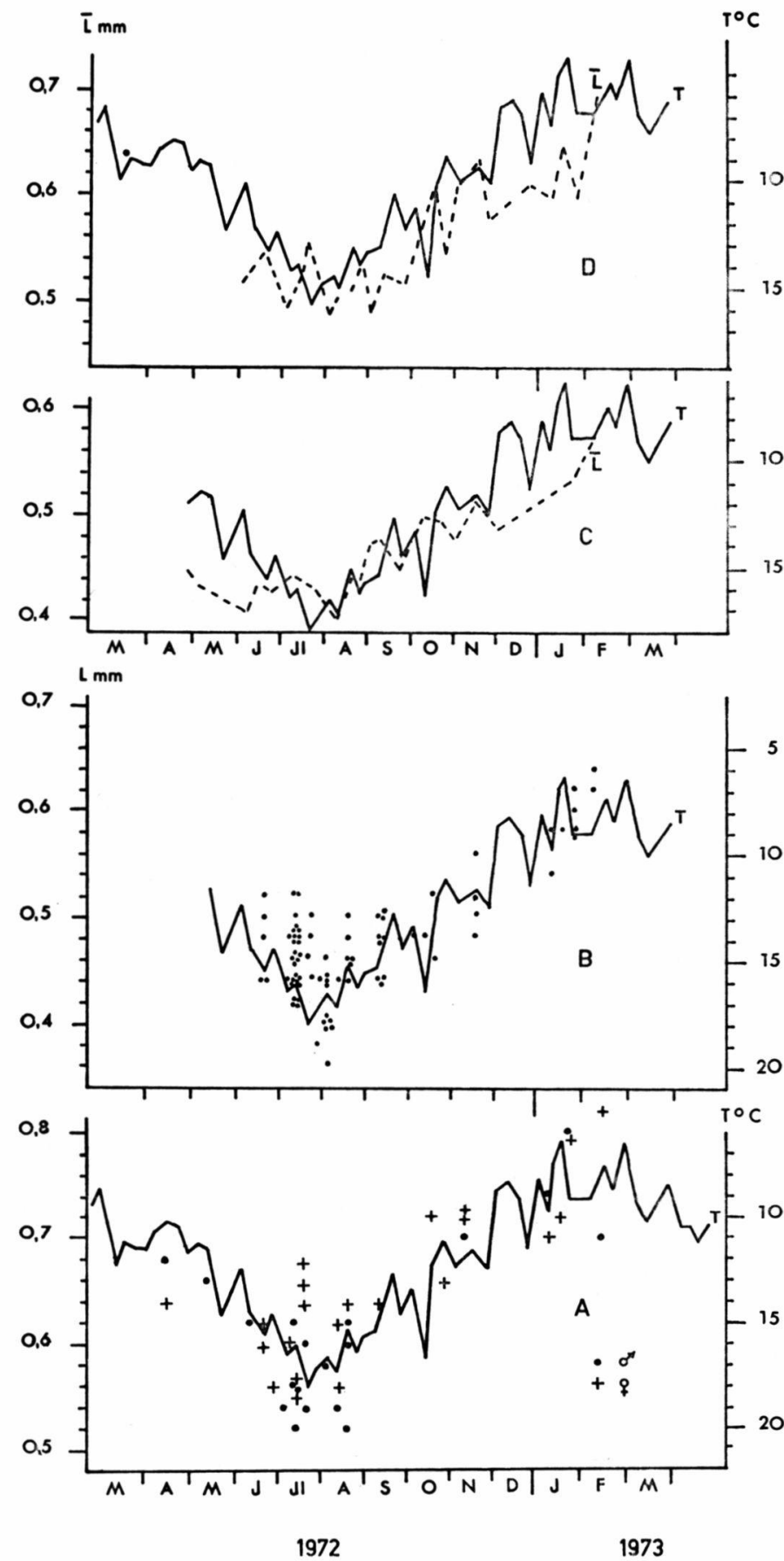

FIG. 6. - Fluctuations saisonnières de la taille des imagos de Simuliidae (L: longueur du basitarse postérieur en $\mathrm{mm} ; \overline{\mathrm{L}}$ : longueur moyenne hebdomadaire $\mathrm{du}$ basitarse postérieur en $\mathrm{mm}$; $\mathrm{T}$ : température moyenne hebdomadaire de l'eau au moment des captures). A) O. ornata $\left(\sigma^{\prime}\right.$ et $\left.\$\right)$; B) $W$. equina et $W$. mediterranea $\left(0^{*}\right)$; C) W. lineata $\left(0^{*}\right)$; D) W. lineata $(\%)$. 
qui correspondent bien aux quatre générations d'adultes (fig. $7 \mathrm{~A}$ ). Les maximums se retrouvent au niveau des derniers stades larvaires, c'est-à-dire des larves qui possèdent des ébauches branchiales nymphales bien sclérifiées (fig. $7 \mathrm{~B}$ ). Par contre, l'apparition des nouvelles cohortes de jeunes stades est moins nette, surtout au niveau du benthos, il y a souvent confusion entre deux générations (fig. $7 \mathrm{C}$ ).
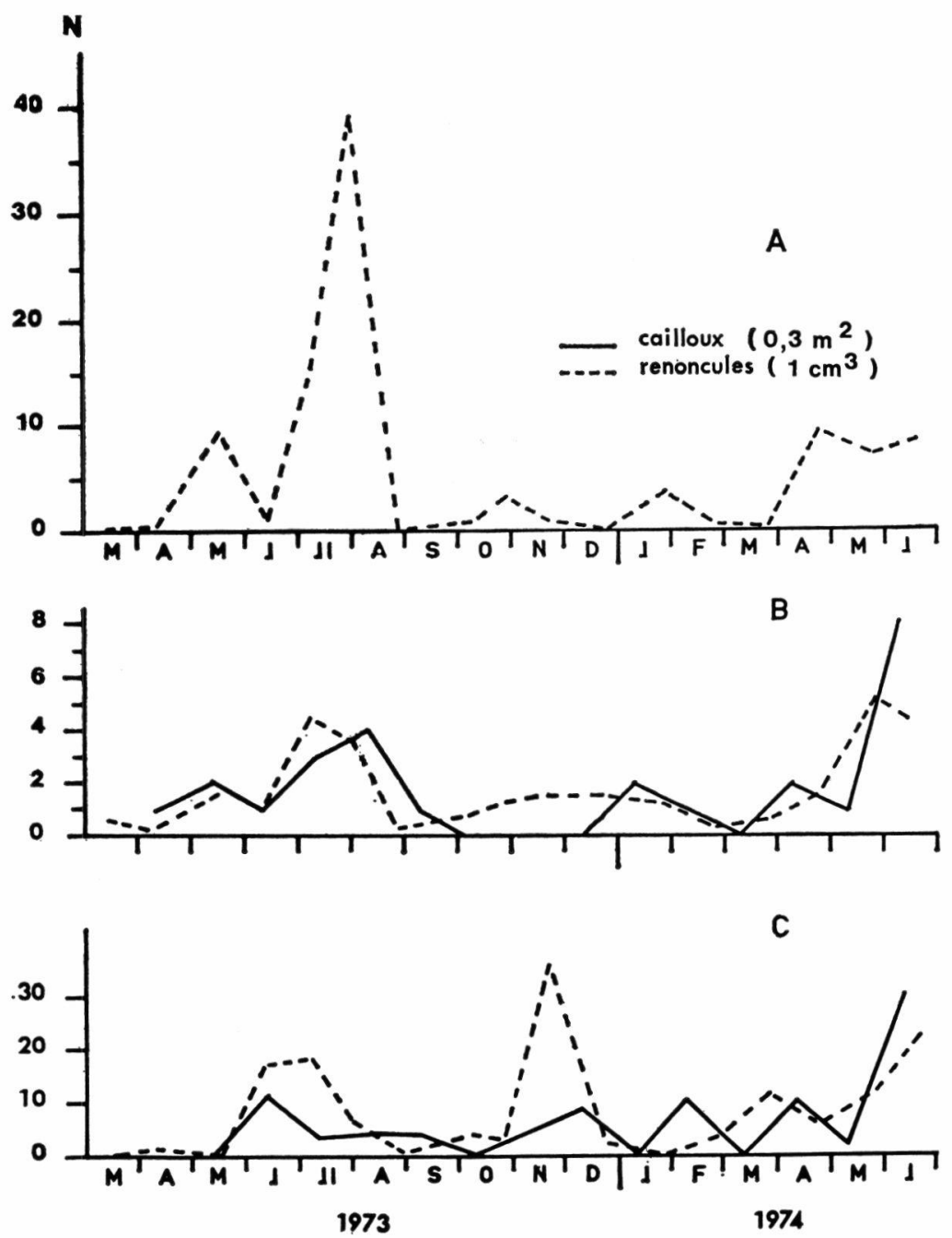

Fig. 7. - Fluctuations numériques des larves et nymphes de Simuliidae au niveau du benthos. A) Nymphes; B) Larves au dernier stade ; C) Jeunes larves (tête inférieure à $0,16 \mathrm{~mm}$ de longueur). 
La détermination spécifique des nymphes est possible et l'on peut suivre l'évolution des quatre espèces dominantes (fig. 8). La synchronisation interspécifique des éclosions se vérifie et l'on remarque la
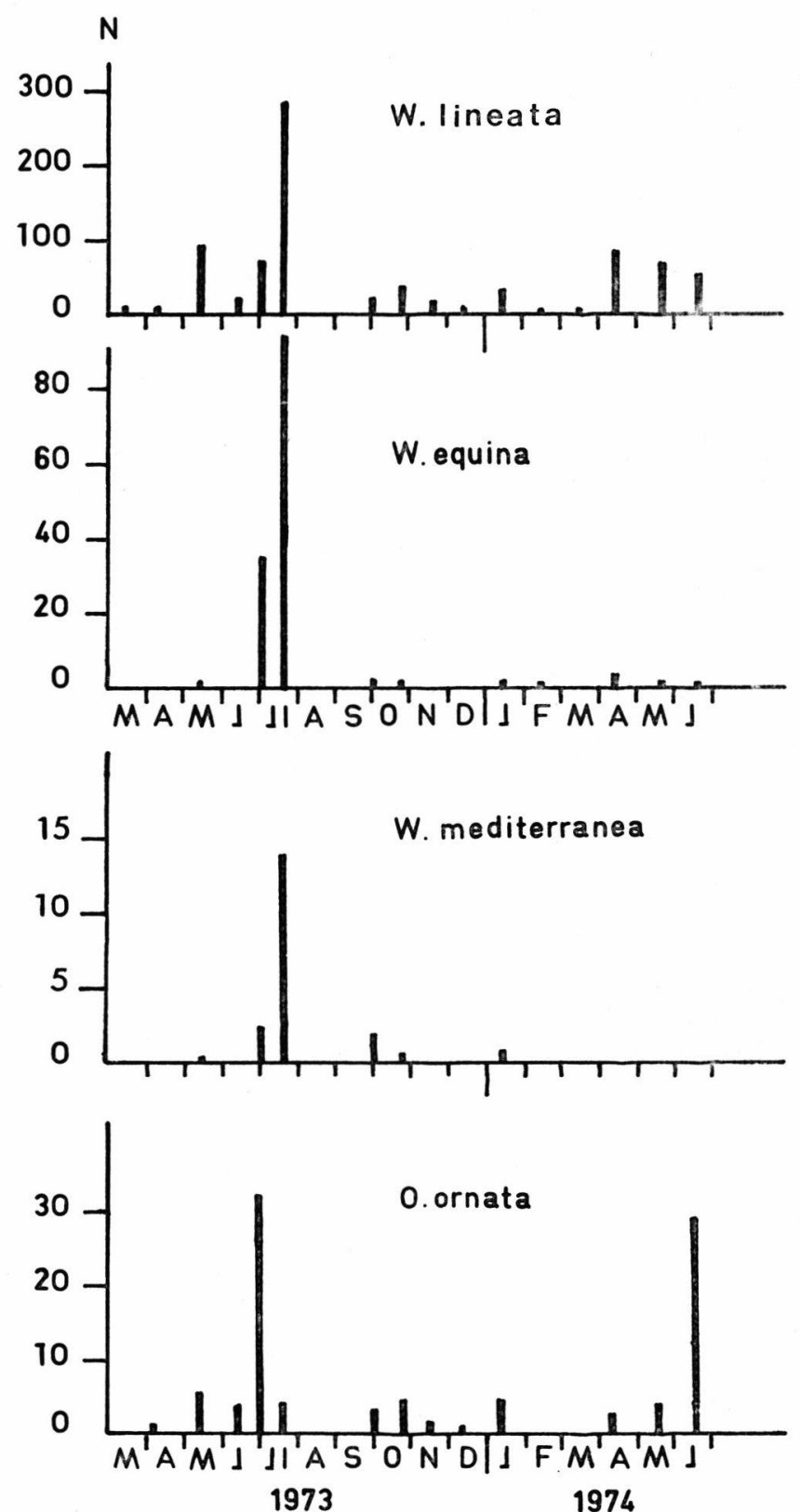

Fig. 8. - Variations mensuelles de la densité des différentes espèces de nymphes dans $10 \mathrm{~cm}^{3}$ de renoncules. 
présence de nymphes de $W$. equina en avril-mai, à un moment où il n'y avait pas d'adultes au piège lumineux. De même, il y a une nymphe de $W$. mediterranea; ceci confirme bien la présence de quatre générations pour ces espèces. L'importance relative de la génération estivale est plus forte chez $W$. equina et $W$. mediterranea que chez $W$. lineata et $O$. ornata.

Dans les renoncules, la densité nymphales $\left(D_{N}\right)$ est en relation avec la densité du dennier stade larvaire $\left(D_{\mathrm{L}}\right)$ :

$$
\mathrm{D}_{\mathrm{N}}=3,46+2,14 \mathrm{D}_{\mathrm{L}}
$$

(liaison hautement significative avec $\mathrm{r}=0,77$ pour 17 couples). Cette supériorité du nombre de nymphes suppose une accumulation relative. Le phénomène n'est pas dû à une stagnation au stade nymphal, celui-ci est en effet court (environ une semaine à $15^{\circ} \mathrm{C}$ ). Il peut s'expliquer par la compétition: les larves conservent une certaine distance entre elles, alors que la passivité nymphale permet un resserrement spatial et une plus forte densité.

\subsection{Les populations larvaires}

L'examen des structures larvaires au cours du temps permet de suivre l'évolution des différentes générations ( $f i g .9$ ).
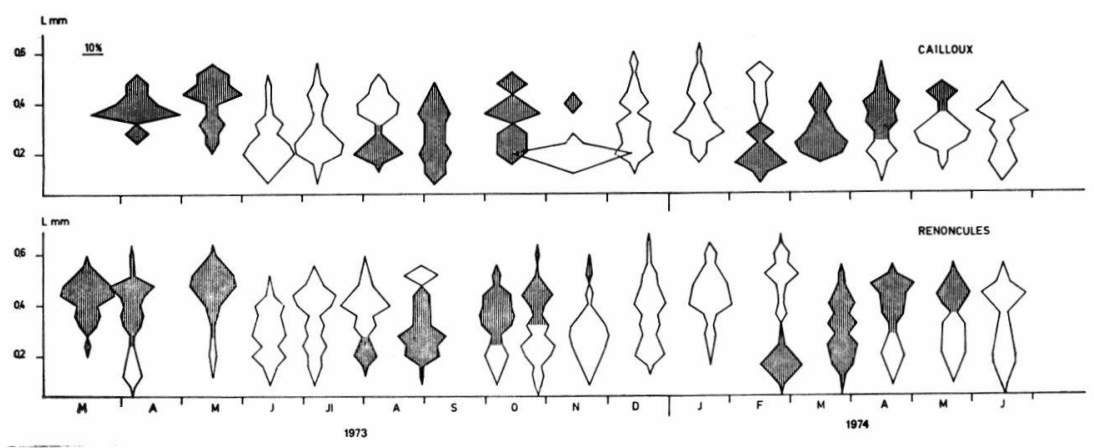

Fig. 9. - Evolution dans le temps des structures larvaires sur les cailloux et les renoncules de 1973 à 1974 ( $\mathrm{L}$ : longueur de la capsule céphalique en $\mathrm{mm}$ ).

L'évolution des structures larvaires tant sur les cailloux que sur les renoncules est comparable; il semble cependant y avoir une distorsion quant à la microdistribution larvaire. Les jeunes stades sont proportionnellement plus abondants sur les cailloux que sur les renoncules, c'est l'inverse pour les derniers stades (fig. $10 \mathrm{~A}$ ). Les différences sont très nettes à certaines époques de l'année, en particulier au moment des basses eaux. Ainsi, en juin 1973, des prélèvements effectués avec le même filet simultanément sur les cailloux, les 
renoncules et les feuilles des macrophytes des berges (Phragmites, graminées, etc.), montrent une microdistribution différente des stades (fig. $10 \mathrm{~B}$ ). Les œufs se trouvent en grande quantité sur les feuilles des plantes rivulaires qui touchent l'eau, ce qui explique l'abondance
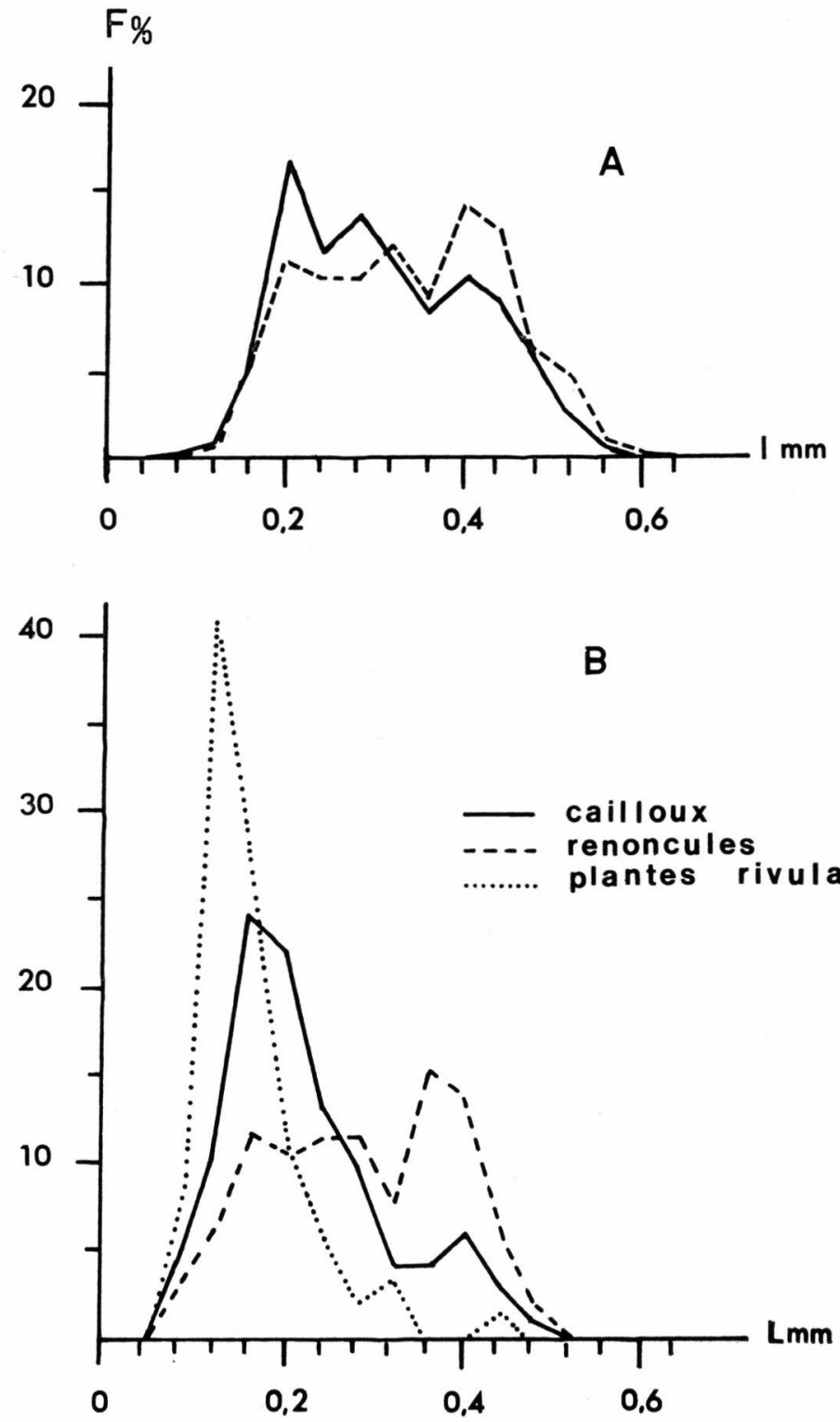

Fig. 10. - Microdistribution des différents stades larvaires (L: longueur de la capsule en mm). A) Moyenne annuelle; B) Juin 1973. 
relative des très jeunes stades, bien que les deux premiers soient mal retenus par le filet. Il y a ensuite une dispersion avec fixation sur les galets et les renoncules.

Les variations saisonnières de la longueur de la capsule céphalique des derniers stades sont un prélude aux variations de taille des imagos (fig. 11).

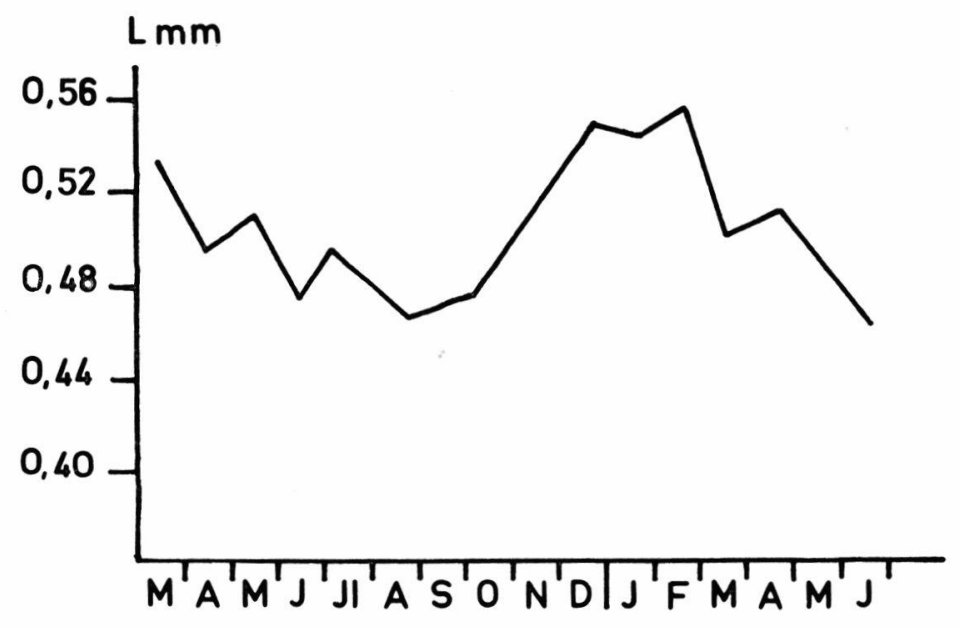

1973

1974

Fig. 11. - Fluctuations saisonnières de la taille du dernier stade larvaire (L : longuer de la capsule céphalique en $\mathrm{mm}$ ).

\subsection{Biomasse et production larvaires}

Des calculs de densité pondérale peuvent être effectués à partir des relations suivantes :

$$
\mathrm{PH}=21,449 \mathrm{~L}^{3,287}(\mathrm{n}=24, \mathrm{r}=0,980)
$$

( $\mathrm{PH}=$ poids humide essoré en $\mathrm{mg}, \mathrm{L}=$ longueur de la capsule céphalique en $\mathrm{mm}$ ).

$$
\text { PS }=0,220 \mathrm{PH}-0,016(\mathrm{n}=7, \mathrm{r}=0,945)
$$

(PS $=$ poids sec après séchage de $24 \mathrm{~h}$ à $60^{\circ} \mathrm{C}$ ).

La densité pondérale suit les mêmes variations que la densité numérique (fig. 12). La biomasse sur les renoncules présente deux maximums : le plus fort en été, un autre en hiver. Il en est pratiquement de même sur les cailloux, mais avec un pic de printemps plus individualisé et une biomasse d'hiver plus forte. Ceci s'explique par l'incidence des crues sur les herbiers.

A biomasse comparable, la génération d'été donne plus d'adultes 


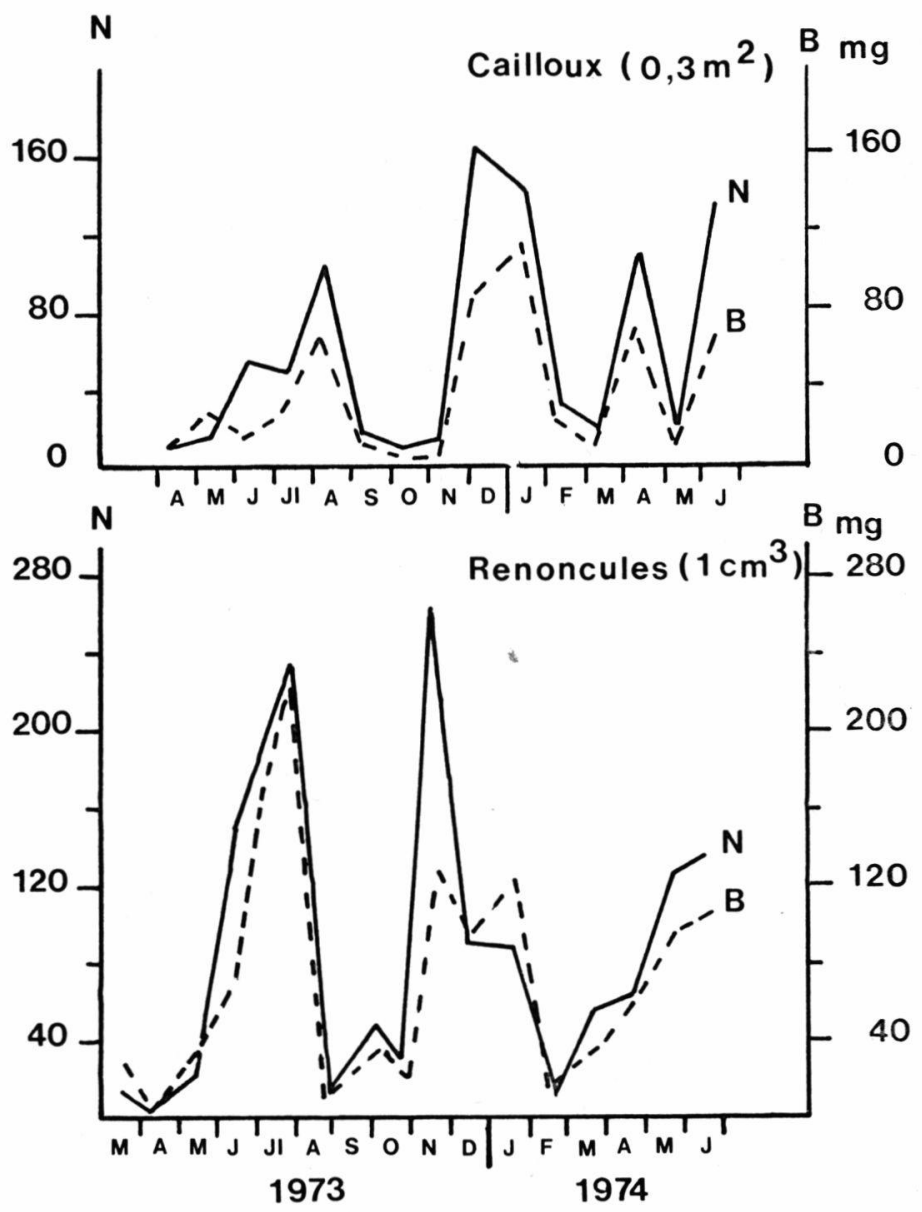

Fig. 12. - Densité numérique (N) et pondérale (B) du peuplement en larves de Simuliidae.

mais nettement plus petits. Les espèces s'adaptent aux variations des conditions du milieu par une adaptation de leur stratégie démographique.

La relation entre la densité sur les cailloux et celle sur les renoncules dépend de la saison. Ainsi d'avril à octobre 1973, en période de croissance maximum des herbiers, l'occupation des renoncules est très forte par rapport à celle des cailloux : $D_{R}=0,32+2,56 D_{v}$ $\left(\mathrm{D}_{\mathrm{R}}=\right.$ densité numérique larvaire pour $1 \mathrm{~cm}^{3}$ de renoncules, $\mathrm{D}_{\mathrm{v}}=$ densité pour $0,3 \mathrm{~m}^{2}$ de cailloux, avec $\mathrm{r}=0,92$, hautement significatif pour 7 couples). Par contre pour la période suivante, la réduction des herbiers par les crues peut inverser les densités, il y a plus de larves sur $0,3 \mathrm{~m}^{2}$ de cailloux que sur $1 \mathrm{~cm}^{3}$ de renoncules ; mais il n'est pas possible de calculer une relation valable. 
A partir des cohortes définies sur la figure 9, il est possible d'avoir une estimation de la production larvaire annuelle dans les deux substrats. Ceci en utilisant trois types de calculs employés sur les simulies du Lissuraga (Neveu $1973 \mathrm{c})$ : méthode de Boysen-Jensen $\left(\mathrm{P}_{1}\right)$, courbe d'Allen $\left(\mathrm{P}_{2}\right)$ et taux de croissance instantané de Rickers $\left(\mathrm{P}_{3}\right)$.

Les résultats obtenus pour les cailloux donnent respectivement en poids humide: $P_{1}=271,4, P_{2}=246,9, P_{3}=283,9$ en $\mathrm{mg} / 0,3 \mathrm{~m}^{2} / \mathrm{an}$, avec des rapports $\frac{P}{\bar{B}}$ de $R_{1}=7,27, R_{2}=6,61, R_{3}=7,60$. La répartition moyenne de cette production entre les générations est de 18 p. 100 pour la génération d'été, 5,7 p. 100 pour l'automne, 59,3 p. 100 pour l'hiver et 17 p. 100 au printemps. Les mêmes calculs effectués dans les renoncules donnent $P_{1}=624,8, P_{2}=613,9, P_{3}=605,4$, en $\mathrm{mg} / \mathrm{cm}^{3} /$ an, avec des rapports $P / \bar{B}: R_{1}=7,52, R_{2}=7,39, R_{: 3}=7,29$. La répartition moyenne intergénération est : été $=41,5$ p. 100 , automne $=10,5$ p. 100 , hiver $=36,8$ p. 100 , printemps $=11,2$ p. 100 . Autrement dit, $1,4 \mathrm{~cm}^{3}$ $\mathrm{d}^{\prime}$ herbier à renoncules produit en moyenne autant que $1 \mathrm{~m}^{2}$ de cailloux. Suivant la densité des herbiers il y a de 1 à 2 litres de renoncules par $\mathrm{m}^{2}$ pour l'été, soit pour cette période une production 1500 à 3000 fois supérieure à celle de $1 \mathrm{~m}^{2}$ de cailloux. Ceci représente une très forte production, car si l'on suppose qu'en moyenne un litre de renoncules recouvre $1 \mathrm{~m}^{2}$, cela représente une moyenne annuelle théorique de $145 \mathrm{~g} / \mathrm{m}^{2}$ de matières sèches.

\subsection{Parasitisme larvaire}

Cette concentration larvaire dans les herbiers est un facteur favorable au développement des épizooties. Deux facteurs régulateurs des populations semblent intervenir: un parasitisme à Nématodes Mermithidae et une microsporidiose.

Ces parasites sont apparemment absents d'août à décembre. Dès janvier, 10 p. 100 des larves sont atteintes par les microsporidies, ce taux est maximum à la fin du printemps avec 35 p. 100 en mai, puis chute rapidement en juin-juillet (1 à 2 p. 100). En ce qui concerne les Mermithidae, 8 p. 100 de la population est atteinte en mars, en avril 10 p. 100 ; il y a ensuite une baisse du taux de parasitisme. Les larves parasitées se trouvent en fait de février à juillet; les nématodes sont à un stade avancé, occupant toute la cavité abdominale. Cette période est sensiblement la même que celle observée par Welch et Rubsov (1965).

Aucune larve n'ayant les deux types de parasites simultanément, le taux maximum d'individus malades est de 30 à 43 p. 100 en avrilmai 1973, mais par contre seulement 7 à 9 pour 100 en 1974 pour la même époque. Le parasitisme reste néanmoins important de la fin de 
l'hiver à la fin du printemps; ceci peut être une des causes des faibles captures de nymphes et d'adultes pour la génération de printemps. La génération d'été est beaucoup moins attaquée malgré une densité larvaire forte, autrement dit le développement de l'épidémie est au moins autant sous la dépendance de facteurs propres aux parasites que la densité de l'hôte. Par ailleurs une certaine forme de pollution organique semble favorable au développement du parasitisme (Carlsson 1963, Glötzel 1973).

\section{6. - CONCLUSIONS}

La composition spécifique du peuplement de Simuliidae de la basse Nivelle est caractéristique de cette zone avec une dominance très nette des Wilhelmia liée à la présence des herbiers à renoncules. W. lineata est en effet signalée dans les zones relativement chaudes, souvent en compagnie de W. equina (Davies 1966, Carlsson 1967, Gagneur 1976). O. ornata, espèce ubiquiste, est signalée avec $W$. equina dans les rivières de plaine, mais toujours en densité nettement plus faible (Grenier 1953, Carlsson 1967, Ladle et al. 1972) ; par contre ce n'est pas le cas du Lot (Gagneur 1976). L'association de ces deux espèces avec $W$. lineata se retrouve dans la Fulda (Zwick 1974), mais $W$. mediterranea, espèce plus méridionale, en est absente. Beaucournu-Saguez (1975 a, b) signale lles trois espèces de Wilhelmia dans le Sud de l'Espagne et quelques rares $W$. mediterranea dans le NordOuest; cette espèce est assez abondante dans le Languredoc (Jarry 1973). La présence de celle-ci dans la Nivelle correspond certainement à une limite de son aire de répartition. W. lineata servirait de transition entre $W$. equina au Nord et $W$. mediterranea au Sud.

Le genre Wilhelmia se développe bien dans ce type de rivière grâce aussi à sa tolérance à une certaine forme de pollution et d'eutrophisation (Carlsson 1967, Rivosecchi et al. 1974, Wichand 1976). Il en est est de même de O. ornata (Glötzel 1973). La Nivelle sur ce point présente une légère pollution organique estivale qui peut être néfaste à d'autres espèces. Par contre le développement massif de ces espèces peut avoir un rôle épurateur de l'eau (Ladle 1972, Ladle et al. 1972, Glötzel 1973, Reisen 1974).

Ladle et al. (1972) ne trouvent que trois générations en Angleterre pour $W$. equina avec une hibernation larvaire et quatre générations pour $O$. ornata. Ces résultats sont proches ide ceux de Zahar (1951), Davies (1966), Maitland et Penney (1967); par contre Zwick (1974) signale la probabilité d'une quatrième génération sur le Fulda pour $W$. equina, mais seulement deux à trois générations pour $W$. lineata. La présence de quatre générations sur la Nivelle pour les Wilhelmia et $O$. ornata s'explique par la position méridionale du ruisseau. 
Les estimations de la production des herbiers à renoncules sont beaucoup plus élevées que celle de Ladle et al. (1972) sur un cours d'eau anglais envahi par ces herbiers. Dans le cas de la Nivelle il peut donc y avoir une certaine concentration larvaire dans ce milieu. Les taux de renouvellement de la biomasse moyenne annuelle sont par contre comparables. Ces calculs restent évidemment approchés dans la mesure où il $\mathrm{y}$ a des migrations intersubstrats et surtout une sélectivité des mailles du filet au niveau des jeunes stades.

En ce qui concerne les taux de parasitisme, ils sont semblables à ceux obtenus par Rivosecchi et al. (1974). Ces parasites peuvent réduire fortement les émergences imaginales certaines années.

Si ces populations simuliennes sont une source non négligeable de nourriture pour les jeunes saumons il ne faut pas oublier que les imagos femelles de $W$. equina, de $W$. lineata et de $O$. ornata, entre autres, sont des insectes hématophages et s'attaquent vivement au bétail.

\section{TRAVAUX CITES}

Beaucournu-Saguez (F.). 1975 a. - Récoltes de Simulies (Diptera, Simuliidae) dans le Sud-Est de l'Espagne. Ann. Soc. ent. Fr. (N. S.), 11 (1) : 73-89.

Beaucournu-Saguez (F.). 1975 b. - Sur quelques Simulies (Diptera, Simuliidae) du Nord-Ouest de 'l'Espagne. Ann. Parasit. hum. comp. 50 (1) : 105-122.

BENECH (V.). 1971. - Les variations saisonnières chez un éphéméroptère polyvoltin (Baetis rhodani Pictet) d'un ruisseau à truites des Pyrénées-Atlantiques, le Lissuraga. Thèse Doct. $3^{c}$ cycle, Fac. Sci. Univ. Orléans, 80 p.

Carlsson (G.). 1967. - Environmental factors influencing blackfly populations.

Davies (L.). 1966. - The taxinomy of British flies (Diptera, Simuliidae). Trans. Roy. Entom. Soc. London, 118 (14) : 413-511.

Davies (L.). 1968. - A key to the British black species of Simuliidae (Diptera) in the larval pupal and adult stages. Freshw. Biol. Assoc. Scie. Publ., n ${ }^{\circ} 24$, $125 \mathrm{p}$.

Doby (J.-M.) et Rault (B.). 1960. - Simulies françaises du groupe monticola: Simulium monticola Friedericks, 1920, S. monticola var. celtica var. nov., S. dorieri n. sp. (Diptères, Simuliides). Bull. Soc. sci. Bretagne, Rennes, $35: 241-257$.

GaGneur (J.). 1976). - Répartition des Simuliidae (Diptères) dans le Lot. Annls Limnol., 12 (3) : 287.298.

Glörzel (R.). 1973. - Populationdynamik und Ernährungsbiologie von Simuliidenlarven in einem mit organischen Abwässern verunreinigten Gebirgsbach. Arch. Hydrobiol., 42, suppl. (3-4) : 406-451.

Grenier (P.). 1953. - Simulidae de France et d'Afrique du Nord. Encyclopédie Entomologique, P. Lechevalier, Paris, 159 p.

JARRY (D.). 1973. - Contributions à l'étude des Simulies du Languedoc-Roussillon. II. De quelques espèces des Pyrénées-Orientales. Ann. Parasit. hum. comp., $48(5)$ : 703-712.

LadLe (M.), Bass (I. A. B) et Jenkins (W. R.). 1972 a. - Studies on production and food consumption by larval Simuliidae (Diptera) of a Chalk Stream. Hydrobiologia, 39 (3) : 429-448. 
LadLe (M.). 1972 b. - Larval Simuliidae as a detritus feeders in Chalk Stream. Mem. Ist. Ital. Idrobiol., 29, suppl. : 429-439.

LaPChIN (L.) et Neveu (A.) (sous presse). - Etude écologique sur la basse Nivelle, petit fleuve côtier des Pyrénées. I. Hydrographie, climatologie et hydrologie.

Maitland (P.S.) et Penney (M. M.). 1967. - The ecology of the Simuliidae in a Scottish river. J. Anim. Ecol., $36: 179-206$.

Neveu (A.). 1973 a. - Le cycle de développement des Simuliidae (Diptera, Nematocera) d'un ruisseau des Pyrénées-Atlantiques, le Lissuraga. Ann. Hydrobiol., 4 (1) : 51-75.

Neveu (A.). 1973 b. - Variations biométriques chez les adultes de quelques espèces de Simuliidae (Diptera, Nematocera). Arch. Zool. exp. gén., 114 : 251-270.

Neveu (A.). 1973 c. - Estimations de la production de populations larvaires du genre Simulium (Diptera, Nematocera). Ann. Hydrobiol., 4 (2) : 183-199.

Neveu (A.) et Vignes (J.-C.) (sous presse). - Etude écologique sur la basse Nivelle, petit fleuve côtire des Pyrénées. II. Le macrobenthos.

REISEN (W.). 1974. - A preliminary evaluation of the influence of Simulium spp. (Diptera, Simuliidae) larval populations on the concentration of total suspended particles. Entomol. News, 85 (9-10) : 275-278.

Rivosecchi (L.), Cavallini (C.), Noccioli (M.) et Rubeca (L.). 1974. - Osservazioni ecologiche sui simulidi (Diptera, Nematocera), del fiume Arrone e degli affluenti del lago di Bracciano. Riv. Parassit., 35 (4) : 331-356.

Thibault (M.). 1971 a. - Ecologie d'un ruisseau à truites des Pyrénées-Atlantiques, le Lissuraga. I. Etude critique du milieu. Ann. Hydrobiol., 2 (2) : 209-239.

Thibault (M.). 1971 b. - Ecologie d'un ruisseau à truites des Pyrénées-Atlantiques, le Lissuraga. II. Les fluctuations thermiques de l'eau; répercussion sur les périodes de sortie et la taille de quelques Ephéméroptères, Plécoptères et Trichoptères. Ann. Hydrobiol., 2 (2) : 241-274.

Tiberghien (G.). 1976. - Ecologie des Helodidae, Elminthidae, Hydraenidae d'un cours d'eau des Pyrénées-Atlantiques : le Lissuraga. Thèse Doct. Univ., Fac. Sci. Univ. Toulouse, 440 p.

Welch (H. E.) et Rubzov (I. A.). 1965. - Mermithides (Nematoda, Mermithidae) parasitic in black flies (Insecta, Simuliidae). I. Taxinomy and bionomics of Gastromermis boophthorae sp. n. Canad. Ent., 97 : 581-596.

Wichard (G.). 1976. - Untersuchungen zur Ókologie von Simuliiden (Diptera, Simuliidae) an organisch belasteten Gewässern. Gewässer und Abwässer, 60/61:35-64.

ZaHAR (A. R.). 1951. - The ecology and distribution of black fllies (Simuliidae) in South East Scotland. J. Anim. Ecol., 20 : 33-62.

ZwICK (H.). 1974. - Faunistich-ökologsiche und taxonomische Untersuchungen an Simuliidae (Diptera) under besonderer Berucksichtigung der Arten der Fulda-Gebietes. Abh. senckenb. naturforsch. Ges., 533 : 1-116. 